\title{
Target Charging, Strong Electromagnetic Pulse Emission and Proton Acceleration from Thin Foils at 10 TW IPPLM Femtosecond Laser Facility
}

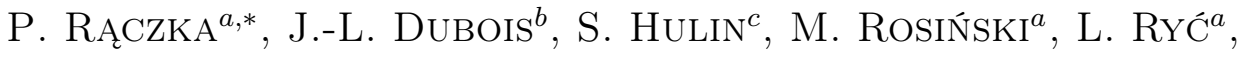

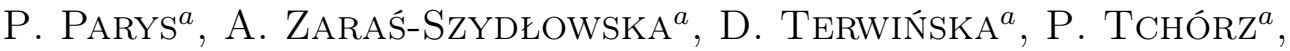

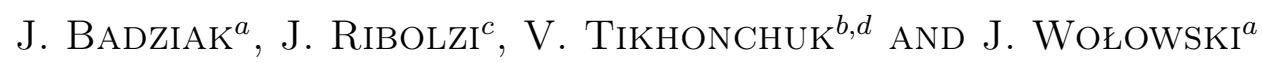 \\ ${ }^{a}$ Institute of Plasma Physics and Laser Microfusion, Hery 23, 01-497 Warsaw, Poland \\ ${ }^{b}$ CELIA, University of Bordeaux-CNRS-CEA, Talence, France \\ ${ }^{c}$ CEA-CESTA, Le Barp, France \\ ${ }^{d}$ ELI-Beamlines, Inst. of Physics, Czech Academy of Sciences, Dolni Břežany, Czech Republic
}

Doi: $10.12693 /$ APhysPolA.138.593

*e-mail: piotr.raczka@ifpilm.pl

\begin{abstract}
It is well known that the laser-target interaction at high power and high laser intensity may result in the emission of strong electromagnetic pulses with frequencies in the range from tens of $\mathrm{MHz}$ to a few $\mathrm{GHz}$ and the duration of hundreds of nanoseconds. It was recently pointed out that the electric polarization of the target and the resulting neutralization current play an important role in the electromagnetic pulses emission. The target charge and the neutralization current generated with laser pulses of $30 \mathrm{fs}$ to several ps duration were studied in detail at the Eclipse laser facility at CELIA, Bordeaux, with the laser pulse energy at the level of up to $100 \mathrm{~mJ}$ on target. In this contribution we report on the measurements of the target charge and the electromagnetic pulses performed at the $10 \mathrm{TW}$ fs laser facility in IPPLM, Warsaw, for the laser pulse energies reaching $400 \mathrm{~mJ}$ on target and the laser pulse duration in the range of 50 fs to $400 \mathrm{fs}$. Due to higher laser pulse energy, the effect of proton acceleration off the rear side of the thin foil targets could be easily observed. This is an important bonus since the mechanism of target normal sheath acceleration of protons which prevails in these laser conditions is closely related to the mechanism of charge ejection from the target, thus providing an additional constraint on any attempts at modeling of the target charge. The collected data is confronted with simplified models of charge ejection and target normal sheath acceleration of protons and qualitative agreement is found.
\end{abstract}

topics: laser-plasma interaction, high intensity lasers, electromagnetic pulses, laser-driven ion acceleration

\section{Introduction}

There has recently been an increased interest in the study of strong electromagnetic pulses (EMP) emitted as a result of laser-target interaction at high power and high intensity laser facilities [1-18]. Such pulses have frequencies in the range from tens of $\mathrm{MHz}$ to a few $\mathrm{GHz}$ and duration of hundreds of nanoseconds. Besides being an interesting physical phenomenon, EMPs are also of considerable practical concern in the forthcoming era of multi-PW laser facilities because they may affect data collection and disturb electronic devices used in experiments. It has recently been emphasized that the charge deposition on target as a result of laser-target interaction and the ensuing neutralization current play a major role in the EMP emission $[3,6]$. Measurements of the neutralization current and the target charge were performed at the Eclipse laser facility at CELIA, Bordeaux, dealing with thick to moderately thin targets $[3,6,17]$. The interest of the IPPLM group is in the target charge deposition on ultrathin $(\mu \mathrm{m}$ scale) targets which are commonly used in experiments involving laser-driven ion acceleration. An experiment with such targets was performed at the Eclipse laser facility in collaboration with the CELIA group, with maximum laser energies on target at the level of $100 \mathrm{~mJ}[14,15]$.

In this paper, we report on the measurement of the target charge and the EMP signal at the $10 \mathrm{TW}$ femtosecond laser facility at the IPPLM, Warsaw, for laser pulse energies reaching $400 \mathrm{~mJ}$ on target. Laser pulse energies at this level resulted in clearly observable laser acceleration of protons. The process of laser acceleration of protons in this regime relies on the same physical mechanisms that are responsible for the charge deposition on the target. Thus, recording the target charge in correlation with the energies of laser accelerated protons, we obtain a data set that allows for a stringent test 
of target charge deposition models. In the following sections, we describe the experimental setup and present preliminary results on the charge deposited on the target as a function of laser pulse energy at fixed pulse duration and laser pulse duration at fixed laser energy. We also present maximum proton energies as a function of the laser pulse energy for fixed pulse duration. We confront this data with two simple models of charge deposition and proton acceleration. Finally, we discuss the electromagnetic field of the strong pulse generated by the lasertarget interaction. Some results on the EMP mitigation which were obtained in this experiment were reported in a previous publication [18].

\section{Experimental setup}

The experiment was conducted at the 10 TW Ti:sapphire laser facility at IPPLM, Warsaw, which delivers a beam with $810 \mathrm{~nm}$ central wavelength and quite good intensity contrast of $5 \times 10^{-9}$. The beam was focused by an off-axis parabolic mirror to a spot of approximately $12 \mu \mathrm{m}$ full width at half maximum (FWHM) and was incident on the target at the angle of $5^{\circ}$ (i.e., the target was rotated relative to the laser beam axis). In this experiment, the laser energy on target was varied between 130 and $400 \mathrm{~mJ}$ and the pulse duration was varied between 49 and 320 fs. The laser pulse energy was measured in each shot using an auxiliary beam leak after the compressor. The uncertainty in the pulse energy is dominated by about $5 \%$ systematic uncertainty in the calibration procedure that is required to correlate the energy of the leak with the energy that is actually deposited on the target. The uncertainty in the pulse duration varied between $0.3 \mathrm{fs}$ for 49 fs pulses to 13 fs for the longest pulses, resulting from the uncertainty in the pulse duration as a function of the compressor setting. For the highest laser parameters in this experiment - $400 \mathrm{~mJ}$ total energy on target and 49 fs pulse duration - the estimated average intensity over the FWHM spot is $2.7 \times 10^{18} \mathrm{~W} / \mathrm{cm}^{2}$, assuming that spatial and temporal beam profiles are Gaussian.

The targets in this experiment were custommade, with $6 \mu \mathrm{m} \mathrm{Al}$ foils pasted over $1 \mathrm{~mm}$ holes drilled in a Cu "pill" $1 \mathrm{~mm}$ thick and $10 \mathrm{~mm}$ in diameter, which in turn was placed in the "lollipop" target holder used in the preceding experiment at the Eclipse laser [14]. The surface of thin foil targets prepared in natural environment is always contaminated with hydrocarbon and water molecules which form an ultrathin (nm scale) layer that becomes the source of protons emitted from the foil upon irradiation by a high-intensity laser. The target holder had the form of a brass ring $14 \mathrm{~mm}$ in diameter placed on a thin brass wire $30 \mathrm{~mm}$ long. It was directly connected via a $50 \Omega$ SMA mount to a coaxial cable which allowed for a direct measurement of the target neutralization current. The SMA mount was attached to an electrically grounded thin
Al plate $160 \times 160 \mathrm{~mm}^{2}$ to ensure the dominant dipole character of the electromagnetic emission of the target holder acting as an antenna.

To monitor the electromagnetic pulses inside the experimental chamber, several probes were used in this experiment but in this paper we only report results obtained with a commercial Prodyn RB230 B-dot probe, coupled with the Prodyn BIB$100 \mathrm{G}$ balun. The RB230 probe was placed $41 \mathrm{~mm}$ above the target, $218 \mathrm{~mm}$ from the target along the incident laser beam direction and $91 \mathrm{~mm}$ to the right from the laser beam in the horizontal plane and was sensitive to the ortho-radial component of the magnetic induction field relative to the vertical axis. This component is expected to be dominant if the main driving process of the electromagnetic pulse emission is the neutralization current in the (vertical) target stalk. Experience from previous experiments for the laser and target parameters similar to ours $[3,6]$ has shown that lasertarget interaction results in a single strong short spike of the return current which generates a single strong electromagnetic pulse that expands into the experimental chamber. This pulse is reflected from the chamber walls and the equipment placed inside the chamber, resulting in decaying electromagnetic oscillations inside the chamber lasting for hundreds of nanoseconds. The shape and magnitude of the strong initial pulse depends on the type of the target and the form of the target support but is independent of the form of the experimental chamber and the distribution of diagnostic equipment inside the chamber. Characteristics of this strong initial pulse are important from the point of view of potential damage to the electric and electronic equipment inside and outside the chamber. On the other hand, the duration and the spectral composition of the decaying oscillations depends strongly on the size and shape of the experimental chamber and on the amount of diagnostic equipment placed inside it. The information about the decaying tail of electromagnetic oscillations is important for reduction of electromagnetic interference in the data collection process; for example, by enlarging the flight path of the recorded ions we may reduce or even completely eliminate the effect of EMP on the readings of solid state ion detectors. Both pieces of information on the EMP signal - the characteristics of the short strong initial pulse and the duration and spectrum of the decaying oscillations - may be meaningfully extracted from the data of even a single electromagnetic probe placed inside the chamber.

To monitor the laser-accelerated ions, a silicon detector was used [19], denoted as FLM. This detector was mounted at a distance of $1863 \mathrm{~mm}$ from the target at the end of a long tube protruding from the experimental chamber exactly along the direction of the incidence of the laser beam. A schematic top view of the experimental chamber is shown in Fig. 1. 


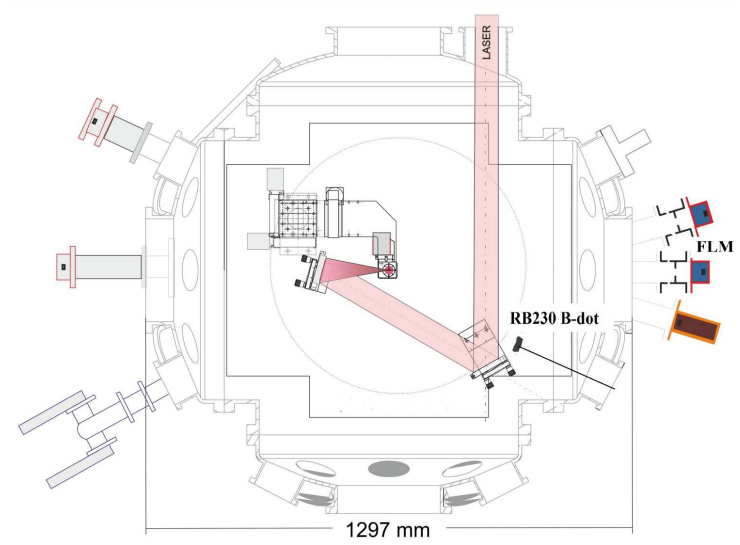

Fig. 1. A schematic view of the experimental setup at the 10 TW IPPLM laser facility. The mounting of the FLM ion detector is not shown in scale - it was actually mounted on a long tube protruding from the experimental chamber.

\section{Measurement of the charge deposited on target}

The total charge deposited on target is extracted from the data on the target neutralization current. The typical neutralization current profile is shown in Fig. 2. It has the form of a narrow spike followed by a few rapidly decaying oscillations, with the basic pulsation lasting no longer than 1 ns. The same form was observed in almost all the shots, with a difference only in the height of the initial spike. This form is almost identical to what was observed in earlier experiments at lower laser energies $[3,14]$ with a small but interesting difference, namely, the presence of a noticeable negative spike just before the main (positive) spike.

By integrating the neutralization current we obtain the target charge as a function of time, from which we can extract the charge deposited in lasertarget interaction by taking a difference of $q(t)$ before and after the main spike (Fig. 3). The charge state after the main spike is estimated as an average of the target charge at the first maximum and first minimum after the main spike. Other prescriptions are possible but it turns out they generate results within $\pm 1 \mathrm{nC}$.

The target charge as a function of the laser pulse energy for fixed pulse duration $\approx 50 \mathrm{fs}$ is shown in Fig. 4. The central line of the linear best fit, namely, $Q=(0.077 \pm 0.005) E_{L}+(-5 \pm 2)$ is shown as well to guide the eye. We find that similarly to previous experiments [3, 6, 14], the target charge displays approximately a linear dependence on the laser pulse energy. The data shows some scatter, particularly for a number of data points obtained for energies very close to $390 \mathrm{~mJ}$, i.e., at the top of the considered laser energy range. Our understanding is that rather than being a manifestation of some complicated physical processes this is a result of random factors, such as inherent

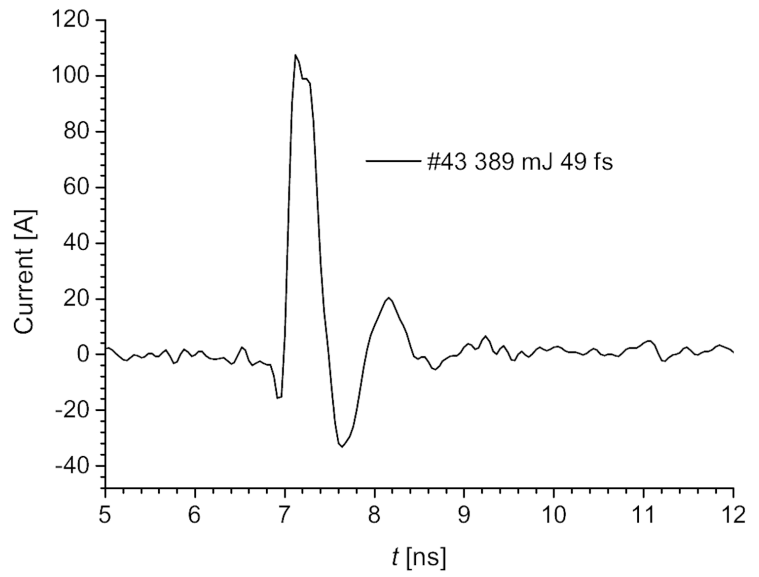

Fig. 2. The temporal dependence of the neutralization current in shot \#43 at the laser pulse energy $389 \mathrm{~mJ}$ and the laser pulse duration $49 \mathrm{fs}$.

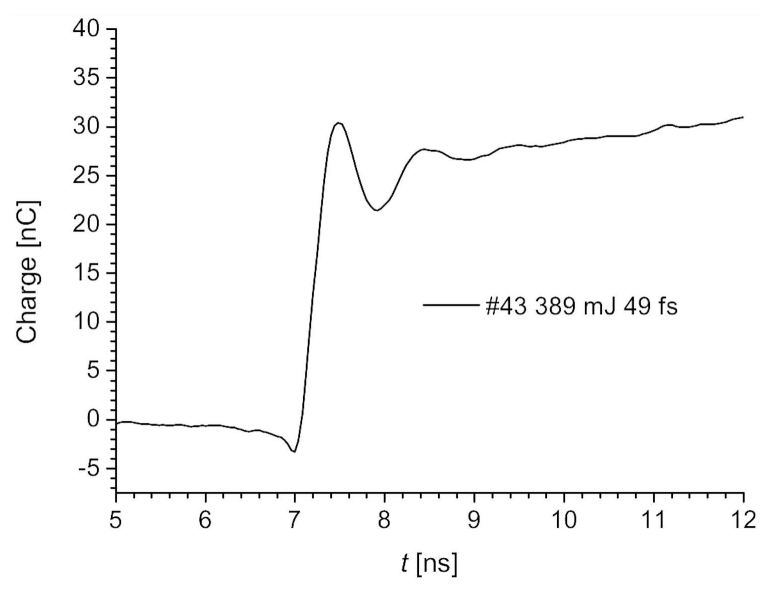

Fig. 3. As in Fig. 2 but for the dependence of the target charge.

fluctuations in the performance of a high-power laser, variations in the target shape, which are unavoidable in custom-made targets, and human inaccuracy, since for each shot the target is brought into focus by manual adjustments. This view is supported by the analysis of the maximum energies of the accelerated protons, discussed further below. It should be noted that despite the fact that the pulse energies involved in this experiment are larger by a factor of 4 , as compared to the previous experiments, the values of the target charge are in fact of similar magnitude. This could be explained by the fact that the laser contrast in this experiment is much better than in the previous experiments and hence there is much less preplasma present on target at the arrival of the main pulse. It was noted in [14] that the target charge appears to be very sensitive to the presence of a preplasma.

The target charge as a function of the laser pulse duration at fixed laser pulse energy is shown in Fig. 5. We find that the dependence is much weaker than it might be expected on the pure scaling of the laser intensity which is consistent with 


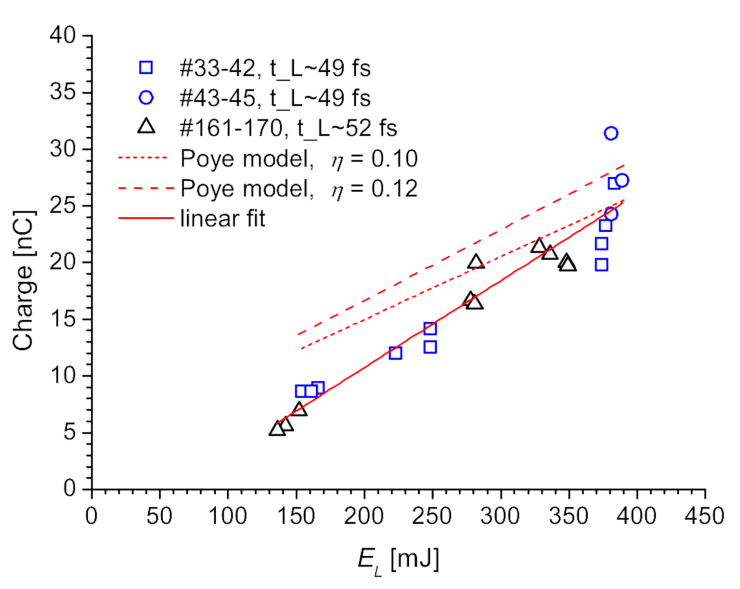

Fig. 4. The target charge as a function of the laser pulse energy, at laser pulse duration $\approx 50 \mathrm{fs}$, compared to predictions of the model by Poyé et al. [17], for two values of the laser absorption coefficient $\eta=0.10,0.12$. Also shown is the line indicating the best linear fit to the data: $Q=(0.077 \pm 0.005) E_{L}+(-5 \pm 2)$.

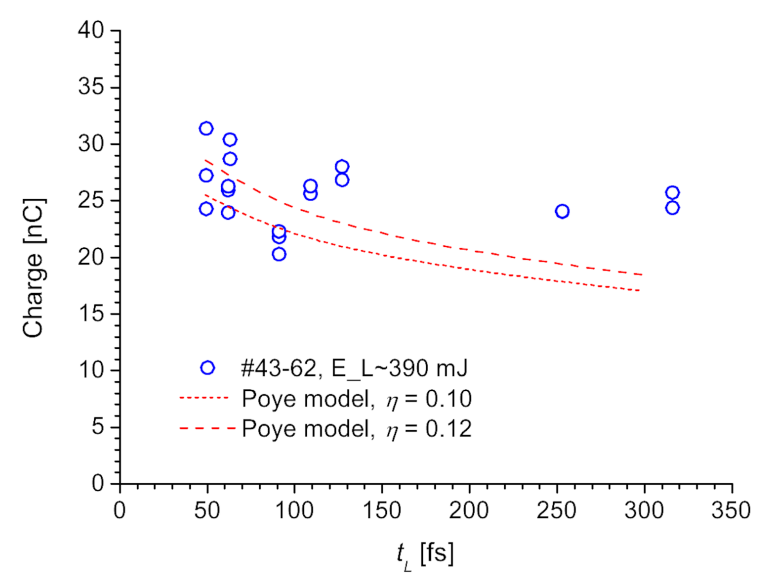

Fig. 5. The target charge as a function of the laser pulse duration at fixed laser pulse energy. The laser energy on target was approximately $390 \mathrm{~mJ}$. For comparison, we show predictions of the model by Poyé et al. [17], for two values of the laser absorption coefficient $\eta=0.10,0.12$.

what was seen in the previous experiments $[3,6,14]$. It should be noted that it is the laser energy before the compressor that was being kept fixed; some fluctuations in the pulse energy after the compressor were observed in this part of the experiment but the data displayed in Fig. 5 is not corrected for this effect. The laser energy on target was approximately $390 \mathrm{~mJ}$.

\section{A simple model of the target charge}

The charging of a laser target is a dynamic process which proceeds with several steps: (i) primary ejection of hot electrons, (ii) formation of the potential barrier at the target surface which confines hot electrons inside the target, (iii) gradual release of hot electrons following the decay of the potential barrier. In principle, such a process could be described by kinetic simulations but in each case this would be a major computational undertaking. For the purpose of quick estimates and parameter optimization, it is of crucial importance to have even a crude but computationally tractable model. An example of such a model appropriate for ultrathin targets was presented in [17]. In this model, the central role is played by the distribution function $f(\varepsilon, t)$ of the hot electrons with the en$\operatorname{ergy} \varepsilon$ inside the target which is assumed to evolve according to:

$$
\begin{aligned}
& \partial_{t} f(\varepsilon, t)=\frac{1}{t_{\text {las }}} f_{\text {las }}(\varepsilon) \theta\left(t_{\text {las }}-t\right) \\
& \quad-\frac{1}{t_{\mathrm{ee}}(\varepsilon)} f(\varepsilon, t)-g_{\mathrm{fr}}(\varepsilon, t)-g_{\mathrm{re}}(\varepsilon, t),
\end{aligned}
$$

where $t_{\text {las }}$ is the laser pulse duration, $t_{e e}$ is the electron cooling time, $g_{f r}$ and $g_{r e}$ are the rates of electron ejection from the front and rear side of the target, and $\theta(t)$ is the step function. It is assumed that part of the laser energy $E_{L}$ is converted into a population of hot electrons, with an exponential distribution in energy $f_{\text {las }}(\varepsilon) \sim \exp \left(-\varepsilon / T_{0}\right)$, where the parameter $T_{0}$ is a known function of the laser intensity and wavelength. The number of hot electrons is $N_{0}=\eta E_{L} / T_{0}$ where $\eta$ is the laser absorption coefficient. The hot electron cloud is assumed to expand with constant velocity $v$ until hot electrons reach their maximum range $r_{\max }(\varepsilon)$, i.e., $t_{\mathrm{ee}}(\varepsilon) \cong r_{\max }(\varepsilon) / v$. They are assumed to occupy a cylindrical region of the target with the radius and depth being simple functions of time and thickness of the target. Parameters of the hot electron distribution are then used to estimate the potential barrier $\Phi(t)$ at the surface of the target, i.e.:

$$
\Phi(t)=\phi_{\mathrm{th}}(t)+\phi_{E}(t),
$$

where $\phi_{\text {th }}$ is the potential due to the negative charge of hot electrons in the Debye layer above the target surface and $\phi_{E}$ is the electrostatic potential due to the positive charge left on the target surface by the escaped hot electrons. The potential barrier determines the minimal energy of the electrons escaping from the target. A numerical implementation of this approach is realized in the Fortran code ChoCoLat2 [17]. The input parameters of the code are the characteristics of the target (material, thickness and radius), characteristics of the laser (the laser wavelength, the size of the laser spot, the pulse energy and duration) and finally the laser absorption coefficient $\eta$. The predictions of the model [17] are compared with the measurements of the target charge in Figs. 4 and 5 for two values of the absorption coefficient $\eta=0.10$ and 0.12 . Agreement is acceptable, although the experiment shows a steeper dependence on the laser pulse energy. This discrepancy is understandable, however, since in reality the absorption coefficient is an increasing function of the laser intensity. The choice of the value of the absorption coefficient requires some 
justification, since this parameter is not very well known, as it depends on fine details of the lasertarget interaction, such as the off-peak pulse temporal profile and the amount of preplasma created on the target surface. However, the same set of parameters, including the laser absorption coefficient, determines predictions for the laser acceleration of protons in the target normal sheath acceleration (TNSA) regime. In fact, the potential barrier that confines hot electrons inside the target is the source of a quasi-static electric field that accelerates protons and carbon ions that are always present in an ultrathin - nanometer size layer of contaminants on the surface of the target. Thus, the information on, say, the maximum proton energies recorded in a given physical situation may be used to constrain the absorption coefficient and other parameters relevant for the target charge and hence substantially reduce the uncertainty in the predictions for the target charge. The arrangement in which the target charge and the proton energies are measured in the same experiment creates favorable conditions for a stringent test of the target charge models.

\section{Data on maximum energies of the laser accelerated protons}

The ions accelerated off the surface of the target were recorded in our experiment using a silicon detector [19] placed in a long tube protruding from the experimental chamber and kept under vacuum. The purpose of using such a tube was to enlarge the flight path of the accelerated ions and hence improve the resolution of the time-of-flight spectra. The accelerated ions consist of protons and various ionization states of carbon ions but the fastest ions are protons. The maximum proton velocities were straightforwardly estimated using the minimal flight time of protons, which is assumed to be equal to the time interval between the photopeak $\left(t_{\text {photo }}\right)$, and the first ion signal $\left(t_{\text {arrival }}\right)$, corrected for the time of travel of the photopeak signal: $t_{\text {min }}=t_{\text {arrival }}-t_{\text {photo }}+L / c$, where $L$ is the distance of the ion detector from the target. Figure 6 shows the maximum proton energies as a function of the laser pulse energy. To guide the eye, the line indicating the best linear fit to the data is also shown: $E_{\max }=(3.6 \pm 0.4) E_{L}+(30 \pm 130)$. The uncertainty in the maximum energy values is dominated by the uncertainty in $t_{\text {arrival }}$ which is determined by the oscilloscope sampling rate and the amount of noise in the ion detector signal. A conservative estimate in our case would be on the order of \pm 2 ns. With the typical value of the time of flight on the order of $120 \mathrm{~ns}$ or more, this implies at worst $4 \%$ uncertainty in the maximum proton energies. The data on maximum proton energies does show some scatter. This scatter is a manifestation of random fluctuations in the laser-target interaction conditions mentioned in Sect. 3, since the phenomenon of laser-driven ion acceleration in this

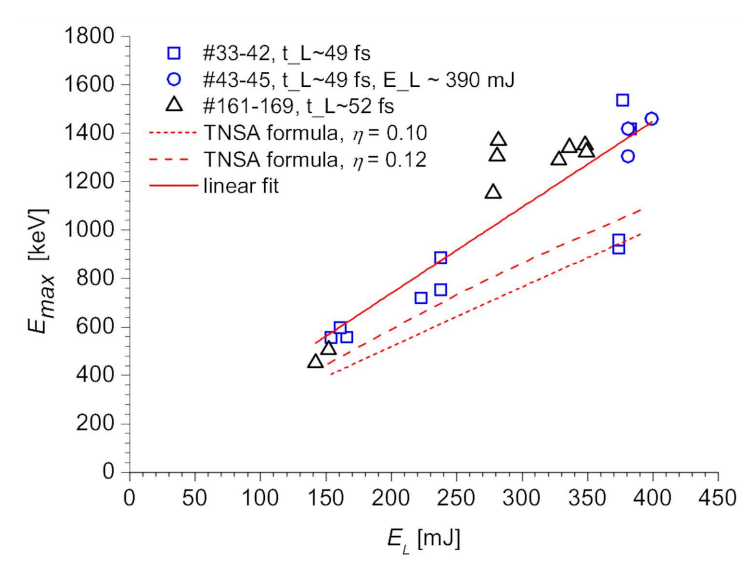

Fig. 6. The maximum proton energies as a function of the laser pulse energy, compared to predictions of the plasma expansion model, for two values of the laser absorption coefficient $\eta=0.10,0.12$. Also shown is the line indicating the best linear fit to the data: $E_{\max }=(3.6 \pm 0.4) E_{L}+(30 \pm 130)$.

laser regime is well-studied and no rapidly changing effects had been identified so far. Furthermore, the scatter in the maximum proton energies appears similar to the pattern displayed by the target charge data which is consistent with the statement that the mechanisms behind these two quantities are closely related and which supports our conclusion that the scatter in the target charge is of random origin and not a manifestation of some complicated physical processes.

\section{A model for the maximum energy of laser accelerated protons}

In order to take advantage of the cross-correlation between the target charge deposition process and the laser proton acceleration we need to confront the data on maximum proton energies with some model. In this paper we shall use a formula inspired by the self-similar, isothermal plasma expansion model [20], which was used in [21] to describe a wide body of ion acceleration data and more recently reviewed in [22]. According to the publications, the cut-off proton energy is given by:

$$
E_{\max }=2 T_{p}\left[\ln \left(t_{p}+\sqrt{t_{p}^{2}+1}\right)\right]^{2},
$$

and the ponderomotive electron energy is defined as:

$$
T_{p}=m_{e} c^{2}\left(\sqrt{1+\frac{I \lambda_{\mu m}^{2}}{1.37 \times 10^{18}}}-1\right),
$$

where $m_{e}$ - the electron mass, $c$ - the velocity of light, $I$ - the laser intensity $\left[\mathrm{W} / \mathrm{cm}^{2}\right]$ and $\lambda_{\mu m}$ is the laser wavelength $[\mu \mathrm{m}]$. The parameter $t_{p}$ is the normalized ion acceleration time:

$$
t_{p}=\frac{\omega_{\mathrm{pi}} t_{\mathrm{acc}}}{\sqrt{2 \exp (1)}}
$$


where $t_{\mathrm{acc}}$ is the effective physical acceleration time and $\omega_{\mathrm{pi}}$ is the ion plasma frequency for hydrogen:

$$
\omega_{\mathrm{pi}}=\sqrt{\frac{e^{2} n_{e 0}}{m_{p} \varepsilon_{0}}},
$$

with $m_{p}$ being the proton mass, $n_{e 0}=$ $N_{0} / c t_{\text {las }} S_{\text {sheath }}$ - the hot electron density, and $S_{\text {sheath }}$ - the area of the sheath at the rear of the target. We have $S_{\text {sheath }}=\pi\left(r_{0}+d \tan \vartheta\right)^{2}$, where $r_{0}$ is the radius of the laser spot on the front side of the target, $d$ is the target thickness and $\vartheta \approx 25^{\circ}$ is the half-divergence angle of the hot electron stream ejected from the front side into the target. However, differently from [21], we assume $t_{\text {acc }}=3\left(t_{\text {las }}+t_{\text {min }}\right)$, where $t_{\text {min }}=60 \mathrm{fs}$, as was recommended in [22]. The absorption coefficient $\eta$ enters this formula through the expression for the number of hot electrons $N_{0}$.

\section{Comparison of model predictions with the data}

In Fig. 6, we show the predictions of this model for $\eta=0.10$ and 0.12 to illustrate the sensitivity of the predictions to this parameter. A practically linear dependence on the laser energy is predicted in our energy range. The data seems to follow a linear dependence, too, albeit with a slightly steeper slope. Similarly as in the case of the target charge model predictions discussed in Sect. 4, this discrepancy in slopes is understandable, because in reality the absorption coefficient is an increasing function of the laser intensity, while we used fixed values for the whole laser energy range considered in this experiment. Predictions with $\eta=0.12$ appear to lie close to the proton data, but the predictions for the target charge for this parameter value lie visibly above the data. The predictions for the target charge are brought close to the data by changing $\eta$ to 0.10 but then the predictions for the maximum proton energies move further below the data. Also in the case of dependence of the target charge on the pulse duration shown in Fig. 5, we see that predictions for $\eta=0.12$ lie closer to the data than for $\eta=0.10$. For both values, however, the predictions run below the data for the longest pulses.

In any case, obtaining an ideal fit with these two models does not seem possible and we did not attempt to further optimize $\eta$ in this approach. It is, however, rather impressive that these two simple models predict values of proton energies and the target charge which are of correct magnitude. Furthermore, they allow us to conclude that a sensible value of the absorption coefficient for our physical situation is around $0.10-0.12$, which is a clear demonstration of the advantages of measuring simultaneously the target charge and the proton energies. It remains an interesting challenge for further work to improve a simultaneous treatment of both these quantities in order to achieve better agreement with the data. In particular, one could use a more advanced model for proton acceleration. We used the formula of [20] because it is simple and explicit, and was found to reproduce the available data with some success [21, 22] but this model may not be the best choice for the rather low laser energies explored in our experiment. Some more advanced models for the TNSA have been reviewed in $[23,24]$.

\section{Measurement of electromagnetic pulses}

The signal $V(t)$ from the RB230 probe is proportional to the derivative of the ortho-radial component of the magnetic induction field $B$ :

$$
V(t)=A_{\text {eq }} \frac{\mathrm{d} B}{\mathrm{~d} t},
$$

where $A_{\text {eq }}$ is the equivalent area of the probe. In order to obtain the reading for $B(t)$, the signal recorded by the oscilloscope is corrected for the attenuation introduced by the balun $(8 \mathrm{~dB})$ and for the external attenuation at the oscilloscope terminal, and then for the frequency-dependent attenuation of the coaxial cable used to connect the probe to the oscilloscope. The resulting data on $\mathrm{d} B / \mathrm{d} t$ is then numerically filtered to the service frequency
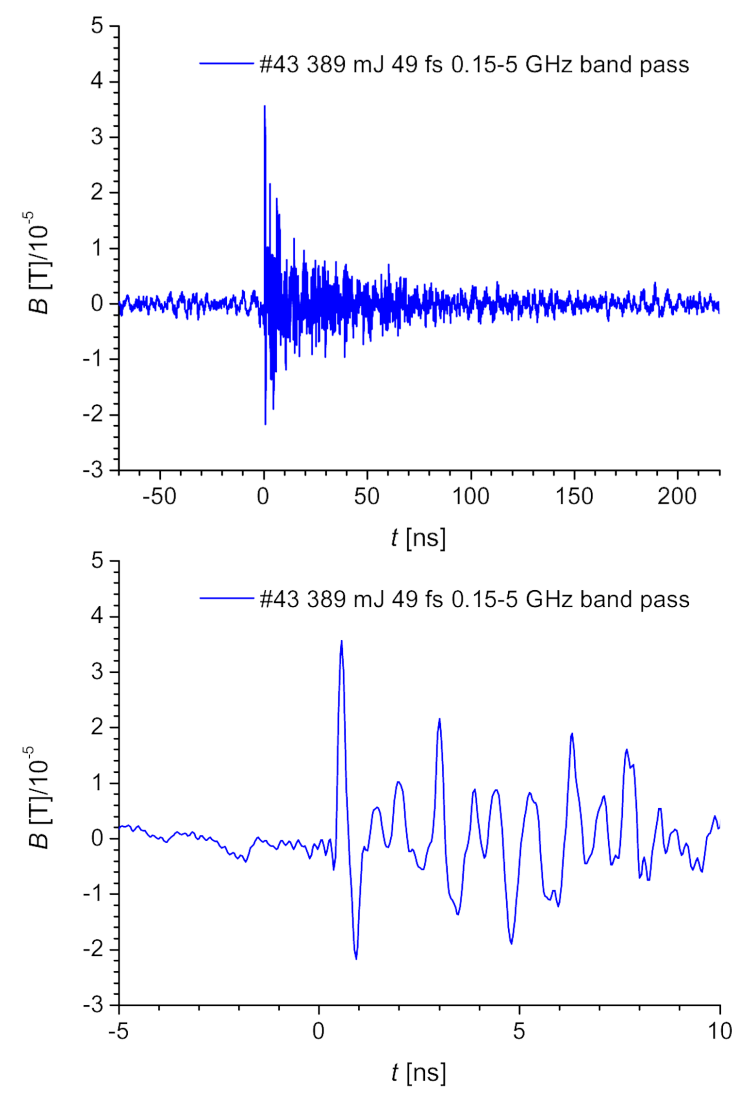

Fig. 7. (a) A typical signal for the ortho-radial component of the magnetic field induction, recorded with the RB230 B-dot probe placed at the distance of $24 \mathrm{~cm}$ from the target. (b) The signal shown in (a) presented on a much shorter time scale to illustrate the fine structure of the initial spike. 


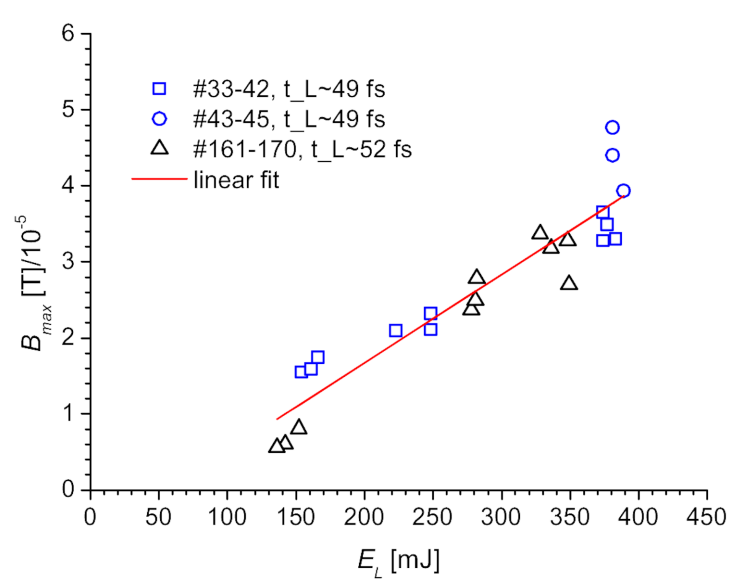

Fig. 8. The maximum value of the ortho-radial component of the magnetic field induction $B$ inside the experimental chamber, at the distance of $24 \mathrm{~cm}$ from the target, as a function of the laser pulse energy $E_{L}$, for laser pulse duration of approximately $50 \mathrm{fs}$. The line of the best linear fit is $B_{\max } / 10^{-5}=(1.15 \pm 0.09) E_{L}+(-0.6 \pm 0.3)$.

range of the $\mathrm{RB} 230$ probe using a $0.15-5.0 \mathrm{GHz}$ band pass FFT filter and finally numerically integrated to yield $B(t)$. A typical $B(t)$ signal is displayed in Fig. 7a. It has the form of a strong initial spike followed by decaying oscillations, lasting in all about $100 \mathrm{~ns}$.

In Fig. 7b, we show the signal presented in Fig. 7a on a much shorter time scale to illustrate the fine structure of the initial spike, the relevance of which was described in Sect. 2. As it can be seen, consistently with the shape of the return current shown in Fig. 2, the initial spike has the form of a single field pulsation lasting $\approx 1$ ns.

Figure 8 presents the maximum value of the measured ortho-radial component of the magnetic induction as a function of the laser pulse energy, for laser pulse duration $\approx 50$ fs. The uncertainty in these values is dominated by the uncertainty in the probe equivalent area which is estimated to be $4 \%$. The data follows approximately a linear dependence on the laser energy, similarly as it was observed in previous experiments of that type $[6,14,15]$. The line of best linear fit is also shown to guide the eye. Values on the order of $5 \times 10^{-5} \mathrm{~T}$ were measured at the top of the energy range (at the distance of $24 \mathrm{~cm}$ from the target). The corresponding values of the electric field strength $E$ may be naively estimated from the relation $E \cong c B$ to be on the order of $15 \mathrm{kV} / \mathrm{m}$.

\section{Summary and conclusions}

We presented data on the target charge for $6 \mu \mathrm{m}$ Al foil, collected at the 10 TW IPPLM laser facility for the laser pulse energies in the range of 130 to $400 \mathrm{~mJ}$ at fixed pulse duration of approximately $50 \mathrm{fs}$ and pulse durations in the range of 49 to 320 fs and the pulse energy on target approximately $390 \mathrm{~mJ}$. The data follows trends observed in previous experiments $[3,6,14,15,17]$, i.e., the energy dependence is approximately linear and there is a weak dependence on the pulse duration. However, despite laser energies higher by a factor of 4, as compared to the previous experiments, the absolute values of the target charge are not significantly higher than those previously observed. That is explained by a smaller laser absorption coefficient in our experiment because of a much better laser contrast at the IPPLM facility and hence a much smaller amount of preplasma present on target at the arrival of the main pulse. The data on the target charge was collected in correlation with the data on maximum energies of the laser-accelerated protons. The processes of target charge deposition and proton acceleration in the TNSA regime are driven by similar physical effects, namely the potential barrier at the target surface and are sensitive to the same parameters. A set of data on these two processes allows for a stringent test of models of charge deposition. We illustrate this using a simple model of the target charge presented in [17] and a simple formula for the maximum energies of laser accelerated protons [20-22]. We show that these very simple models provide predictions in agreement with the obtained data, provided that the laser absorption coefficient is chosen in the range $\eta \approx 0.10-0.12$. This shows that the obtained data set supplies a good ground for a more refined analysis of the target deposition mechanism. Finally, data on the maximum values of the orthoradial component of the magnetic field induction were presented as a function of laser pulse energy, for the pulse duration of $\approx 50 \mathrm{fs}$. Values on the order of $5 \times 10^{-5} \mathrm{~T}$ were measured at the top of the energy range.

\section{Acknowledgments}

The Polish researchers gratefully acknowledge support from the Polish National Science Centre grant Harmonia 2014/14/M/ST7/00024. The French authors acknowledge financial support from the French National Research Agency (ANR) in the framework of "The Investments for the $\mathrm{Fu}$ ture" Programme IdEx Bordeaux-LAPHIA (ANR10-IDEX-03-02). V.T. acknowledges support from the project ELITAS (ELI Tools for Advanced Simulation) CZ.02.1.01/0.0/0.0/16_013/0001793 from the European Regional Development Fund.

\section{References}

[1] C.G. Brown Jr., T.J. Clancy, D.C. Eder, W. Ferguson, A.L. Throop, EPJ Web Conf. 59, 08012 (2013).

[2] F. Consoli, R. De Angelis, P. Andreoli et al., Nucl. Instrum. Methods Phys. Res. A 720, 149 (2013). 
[3] J.-L. Dubois, F. Lubrano-Lavaderci, D. Raffestin et al., Phys. Rev. E 89, 013102 (2014).

[4] M. De Marco, M. Pfeifer, E. Krouský, J. Krása, J. Cikhardt, D. Klir, V. Nassisi, J. Phys. Conf. Ser. 508, 012007 (2014).

[5] J. Cikhardt, J. Krása, M. De Marco et al., Rev. Sci. Instrum. 85, 103507 (2014).

[6] A. Poyé, S. Hulin, M. Bailly-Grandvaux et al., Phys. Rev. E 91, 043106 (2015); erratum Phys. Rev. E 97, 019903 (2018).

[7] F. Consoli, R. De Angelis, P. Andreoli, G. Cristofari, G. Di Giorgio, Phys. Proced. 62, 11 (2015).

[8] F. Consoli, R. De Angelis, L. Duvillaret, P.L. Andreoli, M. Cipriani, G. Cristofari, G. Di Giorgio, F. Ingenitor, C. Verona, Sci. Rep. 6, 27889 (2016).

[9] M. De Marco, J. Krása, J. Cikhardt et al., J. Inst. 11, C06004 (2016).

[10] T. Yi, J. Yang, M. Yang et al., Photonic Sens. 6, 249 (2016).

[11] M. De Marco, J. Krása, J. Cikhardt et al., Phys. Plasma 24, 083103 (2017).

[12] J. Krása, M. De Marco, J. Cikhardt et al., Plasma Phys. Control. Fusion 59, 065007 (2017).

[13] T.S. Robinson, F. Consoli, S. Giltrap et al., Sci. Rep. 7, 983 (2017).

[14] P. Racczka, J.-L. Dubois, S. Hulin, V. Tikhonchuk, M. Rosiński, A. ZaraśSzydłowska, J. Badziak, Laser Part. Beams 35, 677 (2017).
[15] P. Rączka, J.-L. Dubois, S. Hulin, M. Rosiński, A. Zaraś-Szydłowska, J. Badziak, J. Phys. Conf. Series 959, 012005 (2018).

[16] P. Bradford, N.C. Woolsey, G.G. Scott et al., High Power Laser Sci. Eng. 6, e21 (2018).

[17] A. Poyé, S. Hulin, J. Ribolzi, M. GaillyGrandvaux, F. Lubrano-Lavaderci, M. Bardon, D. Raffestin, J.J. Santos, V. Tikhonchuk, Phys. Rev. E 98, 033201 (2018).

[18] J.-L. Dubois, P. Racczka, S. Hulin et al., Rev. Sci. Instrum. 89, 103301 (2018).

[19] L. Ryć, L. Dobrzański, F. Dubecky, S. Jabłoński, P. Parys, W. Słysz, M. Rosiński, Phys. Scr. 91, 074008 (2016).

[20] P. Mora, Phys. Rev. Lett. 90, 185002 (2003).

[21] J. Fuchs, P. Antici, E. D'Humières et al., Nature Phys. 2, 48 (2006).

[22] M. Roth, M. Schollmeier, in: Proc. CASCERN Accelerator School: Plasma Wake Acceleration, Geneva 2014, CERN-2016001, Ed. B. Holzer, CERN, Geneva 2016.

[23] H. Daido, M. Nishiuchi, A. Pirozhkov, Rep. Prog. Phys. 75, 056401 (2012.

[24] A. Macchi, M. Borghesi, M. Passoni, Rev. Mod. Phys. 85, 751 (2013). 